\title{
ENAMEL THICKNESS MEASUREMENTS ON 3D RECONSTRUCTIONS OF TEETH FOR PALEONTOLOGICAL APPLICATIONS
}

\author{
A.V. Gaboutchian ${ }^{1 *}$, V. A. Knyaz ${ }^{2,3}$, E.N. Maschenko ${ }^{4}$, D.V. Korost ${ }^{5}$, A.A. Kudaev ${ }^{5}$ \\ ${ }^{1}$ Peoples’ Friendship University of Russia, 117198, Moscow, Russia - armengaboutchian@ mail.ru \\ 2 State Research Institute of Aviation System (GosNIIAS), 125319 Moscow, Russia - knyaz@gosniias.ru \\ ${ }^{3}$ Moscow Institute of Physics and Technology (MIPT), Dolgoprudny, Russia \\ ${ }^{4}$ Borissiak Paleontological Institute of Russian Academy of Sciences, 117647, Moscow, Russia - evmash@mail.ru \\ ${ }^{5}$ Faculty of Geology, Moscow State University, 119234, Moscow, Russia - dkorost@mail.ru ; a.a.kudaev@gmail.com
}

\section{Commission II, WG II/10}

KEY WORDS: enamel thickness, odontometry, micro-computed tomography, palaeontology, LangTrank cave, Sunghir

\begin{abstract}
:
Findings of teeth play a significant role in palaeoanthropology. And excavations in Vietnamese LangTrank cave serve as a vivid example and evidence of this statement. Teeth constitute the majority of the paleontological material dated to Middle and Late Pleistocene periods. This is to some extent the result of dietary preferences of porcupines as these rodents include in their diets bones of animals however avoiding extremely hard coronal parts of teeth. Under such circumstances teeth serve a key to taxonomic differentiation of findings as genetic analysis is often hindered by a lack of preserved DNA at such dating of material. However morphological analysis is difficult in some cases either, as teeth can be worn out or broken. In that case enamel thickness measurements become an effective study instrument as this feature varies between species. In the current study two teeth with clear signs of expressed dental wear, presumably upper fourth premolars of wild boar required more detailed analysis. Thus they were reconstructed after micro-computed tomography scanning similarly to other upper teeth picked for comparison: orang-utan tooth from the same location and two teeth from the Upper Palaeolithic Sunghir (they have been scanned earlier). This study required new approaches to image processing and measurement methodology due to marked attrition of the samples. The workflow and results of enamel thickness assessments which facilitated taxonomical differentiation of the findings are presented in the article.
\end{abstract}

\section{INTRODUCTION}

Enamel thickness measurements have a well-known historical and methodological background. They serve to shed light on taxonomic differentiation, adaptation and other issues referred to a wide variety of studies in natural science (Alvesalo et al., 2009; Olejniczak et al., 2007; Smith et al., 2009; Zanolli et al., 2017). And notably in paleontological research, when teeth can account for the majority of excavated fossil material (largely due to their durability), the mentioned assessments are of high demand. The obtained measurements results, in line with dental or other morphological studies, are able to facilitate differentiation of the findings. However in some cases, like in the present study, enamel thickness measurements start playing the leading role when teeth, due to their condition, loose their important morphological features.

There are principally two alternative approaches to enamel thickness measurements. One is sectioning teeth and the other requires $\mathrm{x}$-ray application. The first of traditionally implemented techniques is destructive as direct sectioning of studied samples destroys them. Taking into consideration that paleontological findings are unique it would be preferable to preserve them. Regarding conventional planar $\mathrm{x}$-ray imaging, their implementation is limited due to significant distortions in depiction of tooth enamel as its thickness varies across the tooth volume. It should be noted that, in contrast to the mentioned above techniques, microcomputed tomography does not have such disadvantages. Thus a

\footnotetext{
${ }^{*}$ Corresponding author
}

new impetus to enamel thickness measurement has been given with the introduction of $3 \mathrm{~d}$ techniques, especially those based on micro-CT. They enable greater accuracy in reconstruction of external and internal surfaces of enamel. In addition the studied samples' integrity can be completely preserved regardless of numbers and directions of obtained sections.

It should be noted that teeth are difficult object to measure. Of course, there are morphological regularities which are put in the bases of dental measuring techniques. At the same time a lot irregularities, curvatures with complex topography and a large number of varieties exist as well. Under such circumstances results become dependent to a large extent on orientation of the teeth being measured. Hence nearly all studies of sizes of teeth, including enamel thickness measurements, require correct orientation of the measured objects.

Usually expert based traditional approaches are used in odontometric studies. However, orientation of teeth and measurements on contours are a matter of concern and discussions in odontological community (Suwa and Kono, 2005; Zanolli et al., 2010). In anthropological and paleontological research based on $3 \mathrm{D}$ reconstructions from micro-CT scans several approaches are used. Thus enamel thickness measurement can be carried out after orientating tooth by a plane approximated with respect to enamel cervical margin (Olejniczak et al., 2008). As an alternative, dentinal horns or lowest depressions on tooth occlusal surface can serve for orientation purposes as well (Benazzi et al, 2014; Zanolli et al., 2017). According to the mentioned planes 
teeth are sectioned for obtaining contours and conducting measurements on them. Tooth contour analysis usually requires additional constructions based on available and morphologically relevant landmarks (Martin, 1983).

The specific feature of the current study is that the teeth under examination were found to be characterised by very different degree of preservation. The two samples from the LangTrank cave (1t_8 and 1t_9) had a high degree of wear, with their occlusal surface relief having been completely lost its morphologically essential features. Their enamel surface has cracks and chippings involving the area of cervical margins as well. In contrast to these two samples, all the other studied teeth, including the orang-utan upper molar from the LangTrank (o_u) and both upper molars from Sunghir (s_17 and s_27), possess a rather complete morphology. The above-mentioned circumstances, pertaining to the poor condition of some teeth on the one hand and divergent conditions of all samples - on the other, are a hindering factor in the application of the aforementioned techniques that are commonly used.

Thus if we refer to structures necessary for orientation, neither enamel margin nor dentin horns have been preserved on the lt_8 and 1t_9 samples. For this reason development of non-standard approaches to measurements was required. Consequently it has become necessary to pick areas on the teeth studied where the layer of enamel had been preserved in relatively similar condition on all of the studied teeth. Hence an unusual for this kind of studies area of buccal enamel was picked for comparative studies. It should be noted that buccal cervical enamel is not characterised by high stability. These areas relatively often can be affected by carious or non-carious (caused by stress) lesions (Margherita et al., 2017; Nascimento et al., 2016). However, the studied samples possessed intact enamel on the cervical portion of buccal groove. Thus under such conditions of limited choice the research direction was chosen with respect to this uniting all the above-mentioned teeth feature.

A number of our previously conducted studies were held by means of a technique specially developed for measuring teeth - automated digital odontometry (Knyaz et al., 2016, Gaboutchian et al., 2020). Taking into consideration the unusual sample set and study requirements we did not use completely automated measurement and orientation algorithms for the current study.

\section{PALEONTOLOGICAL BACKGROUND}

The studied paleontological dental samples are findings from LangTrank cave situated in the northern Vietnamese Province of Thanh Hoa. It is a multilevel solutional cave in the limestone and dolomite rock body related to the Devonian period. The lowermost eastern galleries of the cave, which were filled at the turn of the Middle and Late Pleistocene periods by loamy red beds with mixture carbonates, contain skeletal and dental remains of mammals (Figure 1). Findings from this location have been studied by palaeontologists and classified as consistent with more than 30 species of large mammals (Lopatin et al., 2019). It should be noted that teeth of an extinct during the Pleistocene period continental species orang-utan (Pongo. sp) in particular have been found in the LangTrank cave. The reason explaining that the majority of findings were represented by teeth of mammal is in habitation of porcupines in that area in Pleistocene (their remains were found as well during the excavations - South-Asian species of Hystrix kiangsenensis Wang, 1931). These rodents include in their diets bones of animals, however avoiding consumption of covered by enamel and for this reason extremely hard coronal parts of teeth.

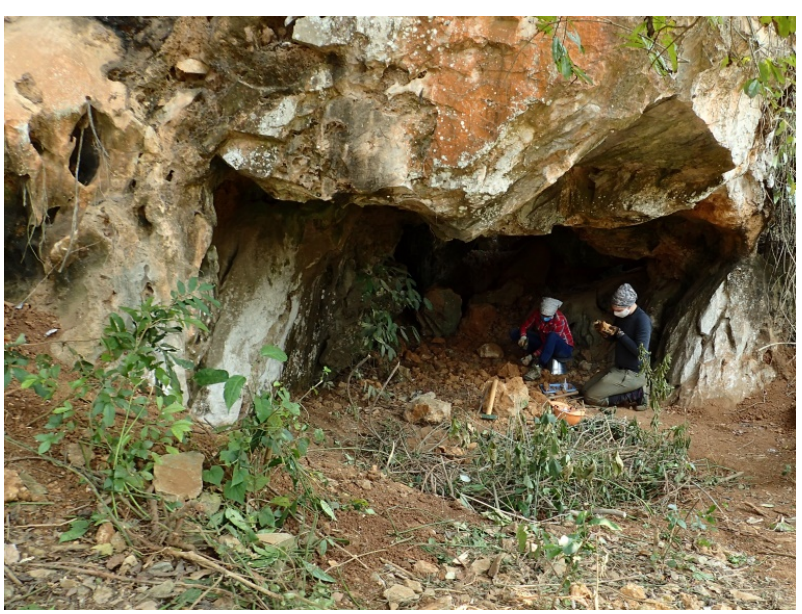

Figure 1. Excavations near the entrance to lower eastern galleries of LangTrank.

One of the most common species of mammals in this location in Pleistocene has been the wild boar (Sus scrofa Linnaeus, 1758). Thus coronal parts of these animals' teeth account for around $25 \%$ of all findings during expeditions. There are teeth which can be referred to animals of different ages: from new-borns with deciduous dentitions to very old specimen with severely worn out distal molars (M3). The majority of difficulties in differentiation and classification of wild boars' dental remains are traditionally related to their upper fourth premolars (P4), especially if their morphology had been changes by the process of functional wear, as their sizes largely correspond to possible morphological variability of human upper molars. Two teeth of that kind, preliminary identified as human, were subjected to currently presented studies (Figure 2a,b). More detailed morphological data has showed that they could exactly refer to boars.

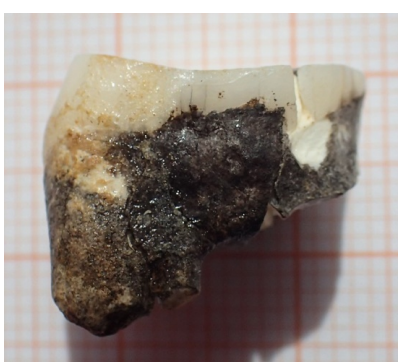

(a)

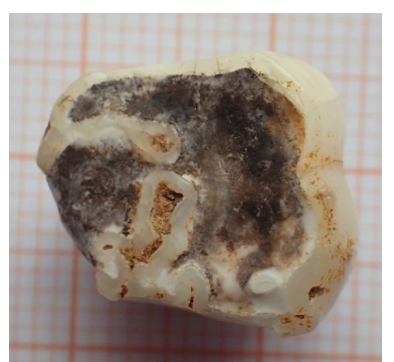

(b)
Figure 2. Studied samples of teeth from LangTrank: 1t_8 view from distal surface (a) and lt_9 view from occlusal surface (b).

Nevertheless this kind of comparative studies of enamel thickness and micromorphology of coronal enamel and dentin surfaces of extinct mammalian teeth is one of the first experiences in terms of the applied methodology as well. Enamel thickness of the studied teeth was assessed in comparison to orang-utan upper tooth (Pongo sp.) from the same location (sample o_u) as well as two upper teeth of an adolescent individual from Upper Palaeolithic Sunghir which is well-known archaeological site situated in Vladimir Oblast, Russian Federation (samples s_17 and s_27).

\section{METHOD}

Software which was applied for the current study has been specially developed at GosNIIAS (Russian Federation). Initially it 
has been used for conducting measurement on digital reconstructions of teeth in a research related to prosthetic dentistry (Knyaz et al., 2007). The software allows uploading 3D images, setting landmark position, different modes of sectioning for contour obtaining and distance measurements on contours. Measurement results can be subsequently saved for further analysis. These procedures run partially in automated mode as well as in "manual" mode.

The process of $3 \mathrm{~d}$ reconstruction obtainment was based on X-Ray imaging through micro-computed tomographic scanning. Samples $\mathrm{s}_{-} 17$ and s_27, being antimeres within a single dental arch, have been previously scanned as a whole with the complete skull on General Electric Phoenix v|tome|x Metrical Edition. Their reconstructions were performed after extraction of their images from the entire stack. Voxel edge for these two human upper second molars accounts for $43 \mu \mathrm{m}$. Samples 1t_8, 1t_9 and o_u as separate teeth (i.e. objects of significantly smaller size than skull) could be scanned in higher resolutions. Thus they were reconstructed after micro-computed tomographic scanning in SkyScan 1172 at $10,3 \mu \mathrm{m}$ inter-slice distance. In both cases $3 \mathrm{~d}$ reconstructions were obtained from images stacks consisting of 8-bit depth .bmp format files.

Further image and reconstruction processing procedures were performed by use of Avizo 9.01 software. Segmentation procedures of the studied objects were based on variety in X-ray absorption by different dental tissues: external highly-mineralised enamel, massive bulk of dentin and internal cavity of pulpal chamber. Areas with same absorption level were sorted and morphologically irrelevant ones were excluded and cleaned. At the final stage models' sizes were reduced in order to allow easy software running and subsequently they were converted to .stl and .x formats.

Enamel thickness measurements were performed on reconstructions of enamel or combined enamel/pulpal chamber. The following procedures provided necessary orientation of slices, sectioning for contour obtaining, setting landmarks and orientation on contours and measurements. On the surfaces of digitally reconstructed enamel models corresponding to their buccal grooves two points were set: the first was localised in the area of intercusp (or inter-root) protrusion of cervical enamel portion and the other - on the projection of buccal groove. As a matter of convenience these points were marked on inner surface of enamel (Fig. 4). The above-mentioned landmark setting is an expertbased "manually" conducted procedure.

Further stages run automatically by means of the used software. The constructions include 10 parallel slicing planes which are perpendicular to line connecting initially set landmarks on the $3 \mathrm{~d}$ reconstruction of the dental enamel. The planes are equally spaced and serve for contour obtaining (Figure 5).

The contours which were obtained and visualised in $2 \mathrm{~d}$ mode, served for enamel thickness measurements in the buccal groove area. Planar geometric constructions combined with measurements were conducted in two stages by means of a properly developed for such studies software tool - a distance measurer operating between two parallel lines. The first construction providing orientation was performed on the external contour of enamel in order to localise the point corresponding the maximal deepening of the groove which would allow precise setting of the subsequent constructions' direction (Figure 5a). This is a manually operated procedure based the following two conditions. The first is singularity of line tangent simultaneously to both (mesial and distal)

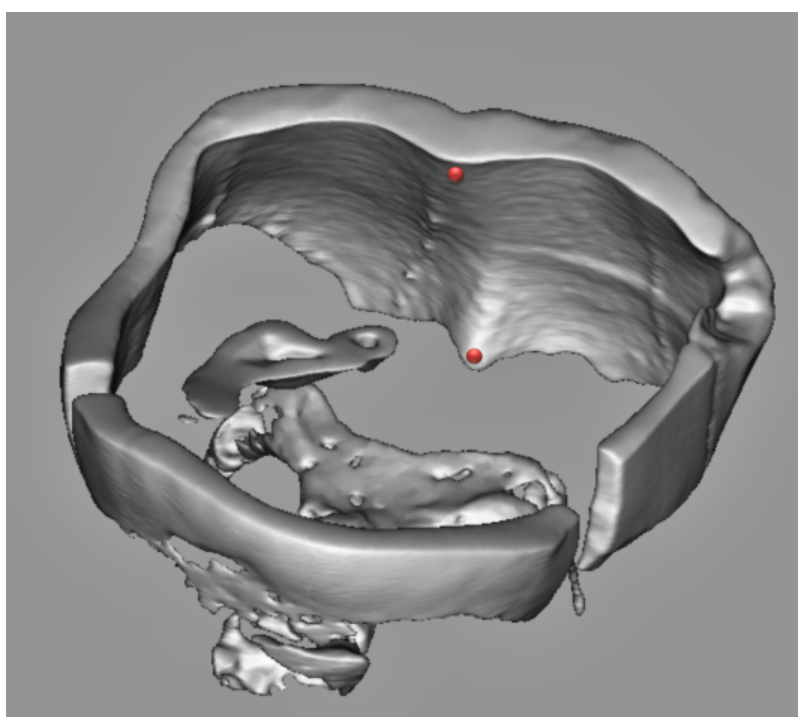

Figure 3. Reconstruction of enamel and pulpal chamber of sample lt_8 with initial landmarks set.

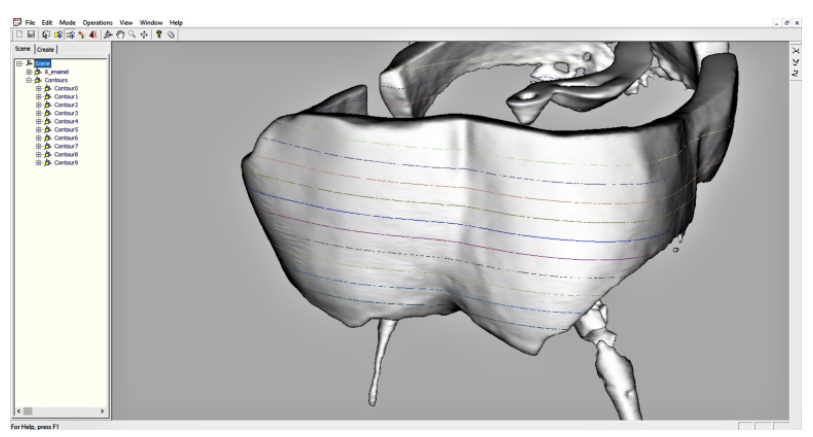

Figure 4. Reconstruction of enamel and pulpal chamber of sample lt_8 with "sliced" contours.

buccal cusp outer surface contours. The second is analogous singularity of another line parallel to the above-mentioned line and simultaneously tangent to the deepest point of the groove contour. The next stage of constructions on $2 \mathrm{~d}$ contours is combined with measurements of enamel thickness. It sets the distance between the two points: groove maximal deepening (localised at the previous stage of constructions) and the most protruded point on the internal, faced to dentin contour of enamel. This point, in its turn, is also localised in compliance with parallelism of lines constructed on the measured contours (Figure 5b). The obtained result corresponds to the required enamel thickness on the current contour and the process is repeated on the next contour.

\section{RESULTS AND DISCUSSION}

The suggested partially automated algorithms including orientational and measuring stages were used for a rather specific research task. At the same time application of ready for use fully automated algorithms has been hampered by the lack on two of the studied teeth of morphologically significant structures. Possibly in the future new algorithms can be suggested which would automatically measure teeth with damaged surfaces as well. However in the current study we had to rely on partially automated methods which possess a certain degree of subjectivity at the initial stage of marking the studied $3 \mathrm{~d}$ reconstructions for orientation. However accuracy and objectivity of measurements has been achieved through combination of the used high-resolution 


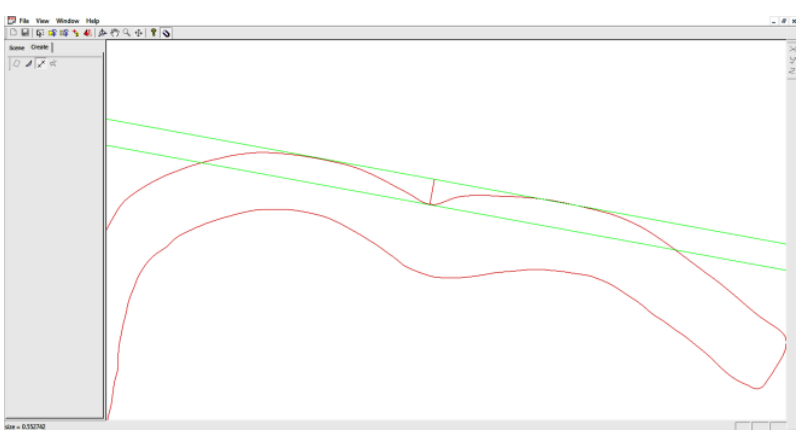

(a) Orientational geometric construction on a sample lt_8 contour

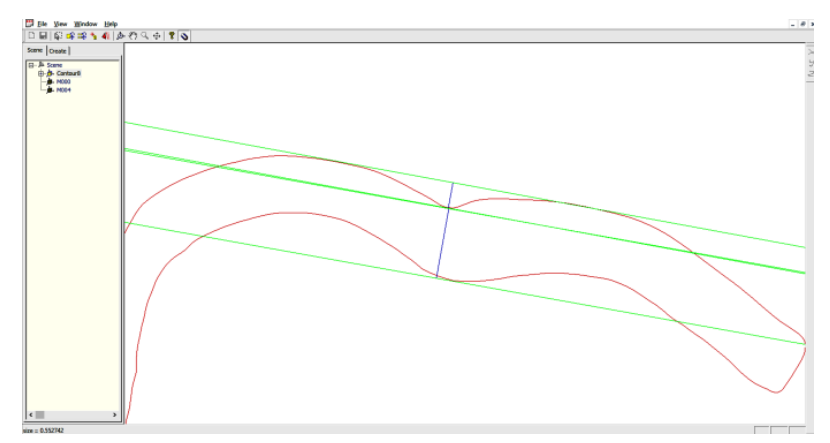

(b) Enamel thickness measurement on a sample lt_8 contour

Figure 5. Orientation algorithms before and after manual adjustments.

imaging technique and the suggested uniform approach to measurements on all of the studied samples. It should mention that enamel thickness varies depending to the localisation of the studied contour. Thus measurements were taken on each of the studied teeth on 10 contours obtained by parallel equally spaced sectioning planes. The results of enamel thickness measurements on the five upper teeth are presented in Table 1.

The samples of teeth which were involved in the current study belong to different species. However even within one individual difference can be found on two antimere teeth. In addition despite the developed measurement technique directed to achieving similarity of measurements on the studied samples, it cannot be expected that the mentioned similarity can become identical. For instance, orientation definitely differs on objects having variety of shapes; and the third contour on any tooth would never correspond by its location to the same contour number on all teeth. This means that there are too many factors to consider a single measurement consistent. This is the reason why average enamel thickness parameters (arithmetic mean of results obtained on 10 contours) are more reliable for assessments and comparisons in such studies. These values referred to each of the studied teeth are presented in the table.

However every measurement is essential as it allows to trace the tendency of changes across the tooth crown. Thus maximal values for each tooth are in highlighted cells as well. Areas with the thinnest enamel layer are located on cervical (closest to roots) portions of dental crowns on all the teeth. Thickness of enamel grows gradually with increasing distance from cervical area towards cusp tips. Maximal enamel thickness values are registered on the most distally located from tooth cervices contours. The only exception is the 1t 9 sample, having the peak of enamel thickness on the 7th contour. Nevertheless this fact does not change the general pattern, as it can be referred to difference of

\begin{tabular}{|c|l|c|c|c|c|}
\hline Contour & \multicolumn{5}{|c|}{ Enamel thickness on contours, mm } \\
\hline & lt_8 & lt_9 & o_u & s_17 & s_27 \\
\hline 1 & 0,676 & 0,379 & 0,112 & 0,282 & 0,233 \\
\hline 2 & 0,905 & 0,574 & 0,123 & 0,497 & 0,421 \\
\hline 3 & 0,981 & 0,888 & 0,394 & 0,562 & 0,518 \\
\hline 4 & 1,018 & 0,89 & 0,506 & 0,759 & 0,661 \\
\hline 5 & 1,085 & 0,969 & 0,559 & 0,939 & 0,895 \\
\hline 6 & 1,207 & 1,052 & 0,582 & 1,052 & 1,187 \\
\hline 7 & 1,346 & 1,142 & 0,645 & 1,303 & 1,387 \\
\hline 8 & 1,499 & 1,119 & 0,591 & 1,474 & 1,508 \\
\hline 9 & 1,541 & 1,12 & 0,786 & 1,64 & 1,708 \\
\hline 10 & 1,598 & 1,08 & 1,011 & 1,728 & 1,863 \\
\hline \multicolumn{7}{|c|}{ Average enamel thickness , mm } \\
\hline \multicolumn{7}{|c|}{1,186} & 0,92 & 0,53 & 1,024 & 1,038 \\
\hline
\end{tabular}

Table 1. Parameters of enamel thickness; individually maximal values are in grey-shaded cells.

dental wear degree on the two (1t_8 and 1t_9) teeth. The other factor that could determine such patters can depend on orientational landmark setting as their positions are determined by inclination of buccal surface which is an individual feature on every separate tooth.

If we summarise the results of dental enamel thickness measurements we can define that the thinnest enamel layer has the $\mathrm{o}_{-} \mathrm{u}$ sample which the upper molar of orang-utan from Plectocene layers of the LangTrank cave. High degree of similarity of enamel thickness patterns is characteristic for antimeres from Sungirian upper dental arch (s_17 and s_27 samples). These human teeth have more bulky enamel in average if compared with the pongine molar as well as highest maximal values on separate sections among all of the studied teeth. The most significant differences can be marked between samples 1t_8 and 1t_9. The first of these two teeth has the highest among the studied samples average enamel thickness. In addition its layer of enamel in the cervical area significantly thicker than on 1t_8, however thick cervical enamel is uniting feature for these two teeth.

The presented measurement and enamel thickness analysis results were used for further studies of paleontological findings and taxonomical interpretations.

\section{CONCLUSION}

The applied in the current study imaging and image processing techniques significantly facilitate for increasing research objectivity, and provide important data in terms of accuracy and detailed visualisation. 3D reconstructions of the teeth allowed to develop and use new non-invasive methods and to receive reliable and as close as possible to objective data on enamel thickness. Such information serves as an important additional support, in line with other traditional morphological assessment methods, in taxonomic differentiation of paleontological findings, especially in cases of controversy. This study is an encouraging example of combining $3 \mathrm{~d}$ imaging and image processing techniques with dental metrics and their implementation in paleontological research, which has a potential for further development.

\section{ACKNOWLEDGEMENTS}

We would like to express our gratitude to the Shared Research Facilities "Palaeoantropological collections Institute of Ethnology and Anthropology of Russian Academy of Sciences" and 
Russian-Vietnamese Scientific Centre for providing the study materials.

\section{REFERENCES}

Alvesalo et al., 2009. Alvesalo, L., Tammisalo, E., Hakola, P. Enamel thickness in 47, XYY males' permanent teeth. Annals of Human Biology - ANN HUM BIOL. 12. 421-427. 10.1080/03014468500007981.

Benazzi et al., 2014. Benazzi, S., D. Panetta, C. Fornai, M. Toussaint, G. Gruppioni, and J. Hublin (2014). Technical Note: Guidelines for the Digital Computation of 2D and 3D Enamel Thickness in Hominoid Teeth. American Journal of Physical Anthropology, 153: 305-313

Gaboutchian et al., 2020. Gaboutchian, A. V., Knyaz, V. A., Novikov, M. M., Vasilyev, S. V., Leybova, N. A., Korost, D. V., Cherebylo, S. A., and Kudaev, A. A.: Automated Digital Odontometry: Measurement Data Analyses In Cases Of Complicated Dental Morphology, Int. Arch. Photogramm. Remote Sens. Spatial Inf. Sci., XLIII-B2-2020, 851-856, https://doi.org/10.5194/isprs-archives-XLIII-B2-2020851-2020, 2020

Knyaz et al., 2007. Knyaz V., Zheltov S., Gabuchyan A., Bolshakov G. Photogrammetric system for automated teeth arches 3D models generation and teeth occlusion analysis. Optical 3D Measurement Techniques VIII, Zurich, 2007, Vol. I, pp. 299-304

Knyaz et al., 2016. Knyaz V. A. and Gaboutchian, A. V.: PHOTOGRAMMETRY-BASED AUTOMATED MEASUREMENTS FOR TOOTH SHAPE AND OCCLUSION ANALYSIS, Int. Arch. Photogramm. Remote Sens. Spatial Inf. Sci., XLI-B5, 849-855, https://doi.org/10.5194/isprs-archives-XLIB5-849-2016, 2016.

Lopatin et al., 2017. Lopatin A.V., Maschenko E.N., Vislobokova I.A., Serdyuk N.V., Dac L.X. Pleitocene Mammals from Lang Trang Cave (Vietnam): New Data. Doklady Akademii Nauk. Nauki o Zhizni. 2021. Vol. 496, pp 5-9, doi: 10.31857/s2686738921010170 (in Russian)

Margherita et al., 2017. Margherita, C., Oxilia, G., Barbi, V., Panetta, D., Hublin, J-J., Lordkipanidze, D., Meshveliani, T., Jakeli, N., Matskevich, Z., Bar-Yosef, O., Belfer-Cohen, A., Pinhasi, R., Benazzi, S. News and Views Morphological description and morphometric analyses of the Upper Palaeolithic human remains from Dzudzuana and Satsurblia caves, western Georgia. Journal of Human Evolution. 113. 10.1016/j.jhevol.2017.07.011.

Martin, 1983. Martin L.B. The relationships of the later Miocene Hominoidea. PhD Thesis, University College London, London. 1983

Nascimento et al., 2016. Nascimento, M., Dilbone, D., Pereira, P., Geraldeli, S., Delgado, A., Duarte, W. (2016). Abfraction lesions: Etiology, diagnosis, and treatment options. Clinical, Cosmetic and Investigational Dentistry. 8. 79. 10.2147/CCIDE.S63465

Olejniczak et al., 2007. Olejniczak, A., Grine, F., Martin, L. Micro-computed tomography of primate molars: Methodological aspects of three-dimensional data collection. Vertebrate Paleobiology and Paleoanthropology. 01/2007, doi: 10.1007/978-14020-5845-5_7

Olejniczak et al., 2008. Olejniczak, A., Smith, T., Feeney, R., Macchiarelli, R., Mazurier, A., Bondioli, L., Rosas, A., Fortea, J., Rasilla, M., Garcia-Tabernero, A., Radovcić, J., Skinner, M., Toussaint, M., Hublin, J-J. Dental tissue proportions and enamel thickness in Neandertal and modern human molars. Journal of human evolution. 55. 12-23. 10.1016/j.jhevol.2007.11.004.

Smith et al., 2009. Smith, T., Olejniczak, A., Kupczik, K., Lazzari, V., Vos, J., Kullmer, O., Schrenk, F., Hublin, J-J., TEUKU, J., Tafforeau, P. Taxonomic assessment of the Trinil molars using non-destructive 3D structural and development analysis. PaleoAnthropology. 2009. 117-129.

Suwa and Kono, 2005. Suwa, G., Kono R.T. A Micro-CT Based Study of Linear Enamel Thickness in the Mesial Cusp Section of Human Molars: Reevaluation of Methodology and Assessment of Within-Tooth, Serial, and Individual Variation. Anthropological Science, 113: 273-289

Zanolli et al., 2010. Zanolli, C., Bayle, P., Macchiarelli, R. Tissue proportions and enamel thickness distribution in the early Middle Pleistocene human deciduous molars from Tighenif, Algeria. C. R. Palevol 9 (2010) 341-348. doi:10.1016/j.crpv.2010.07.019

Zanolli et al., 2017. Zanolli C., Bayle, P., Bondioli, L., Dean, M., Le Luyer, M., Mazurier, A., Morita, W., Macchiarelli, R. Is the deciduous/permanent molar enamel thickness ratio a taxonspecific indicator in extant and extinct hominids? Comptes Rendus Palevol. 16. 10.1016/j.crpv.2017.05.002. 